congenital anomalies, late-onset sepsis (LOS) (57.8\% vs. $23.8 \%$ OR:4.40; 95\%CI:4.0-4.84) and symptomatic PDA (56.0 vs. $30.6 \%$; OR:2.9; 95\%CI:(2.6-3.2)). After adjusting for all BPD predictive perinatal risk factors (BW, GA, Apgar scores, gender and congenital anomalies (AUC:0.8, 95\%CI:0.79-0.81), the factors strongly associated with BPD, other than BW and GA, were LOS (OR:2.54; 95\% CI:2. 27-2.83) and symptomatic PDA (OR:1.54; 95\%CI:1.38-1.73).

Conclusion In this large cohort of VLBW/VLGA, the rate of BPD was $16 \%$ (15.4-16.1\%), strongly associated with GA and BW but also with LOS and symptomatic PDA.

Acknowledgements We thank patients and participating NICU's EuroNeoNet is supported by the DGSANCO funded project EuroNeoStat (No. 2008-1311).

\section{EARLY PREDICTION OF BRONCHOPULMONARY DYSPLASIA (BPD) BY AN EASILY AVAILABLE RISK SCORE}

doi:10.1136/archdischild-2012-302724.0596

'M Roth-Kleiner, ${ }^{1} \mathrm{~J}$ Chnayna, ${ }^{1} \mathrm{E}$ Giannoni, ${ }^{2} \mathrm{M}$ Faouzi. ' ${ }^{1}$ Clinic of Neonatology, University Hospital of Lausanne, CHUV; ${ }^{2}$ Center of Clinical Epidemiology, Institute of Social and Preventive Medicine, University of Lausanne, Lausanne, Switzerland

Background Early prediction of BPD is important for identifying high risk patients likely to benefit from preventive treatment approaches and for providing prognostic information. Therefore we aimed to develop a risk score for BPD based on early available clinical parameters.

Patients and methods: All infants born at the University Hospital of Lausanne < 32 weeks of gestation (WG) between 1998 and 2007 $(n=936)$ were included. Patients diagnosed with RDS $(n=232)$ were divided in two groups, either developing BPD or not. Independent risk factors for the development of BPD were searched by multivariate logistic regression analysis. The $\beta$-coefficients $(\beta=\log (\mathrm{OR}))$ derived from the fitted multivariate model were used to build a scoring system. An internal validation was performed using a twofold cross-validation technique with two subgroups: two thirds of the patients were used as training set for model calibration and one third as prediction set.

Results BPD-risk score was developed based on five covariates: intubation in the delivery room, early neonatal infection, duration of invasive mechanical ventilation in days, birth weight and gestational age, weighted according their $\beta$-coefficients. Area under curve (AUC) was 0.896 . Sensitivity and specificity reached $82.7 \%$ and $82.6 \%$ with a score cut-off of -8 (range -25 to +17 ). Internal calibration proved a good prediction: AUC for the same cut-off was 0.882 for the training set and 0.927 for the prediction set.

Conclusions A simple scoring system available within the first postnatal week can reliably predict the probability of developing $\mathrm{BPD}$ in infants born $<32 \mathrm{WC}$.

\section{VALPROIC ACID-MEDIATED PROTECTION AGAINST HYPEROXIC LUNG INJURY VIA HISTONE DEACETYLASE INHIBITION IN A NEONATAL RAT MODEL}

doi:10.1136/archdischild-2012-302724.0597

${ }^{1} \mathrm{M}$ Cetinkaya, ${ }^{2} \mathrm{M}$ Cansev, ${ }^{1} \mathrm{~F}$ Cekmez, ${ }^{1} \mathrm{C}$ Tayman, ' $\mathrm{FE}$ Canpolat, ${ }^{2} \mathrm{IM}$ Kafa, ${ }^{2} \mathrm{E}$ Orenlili, ${ }^{3} S$ Uysal, 'SU Sarıcı. 'GATA Teaching Hospital, Ankara; 'Uludağ University Medical Faculty, Bursa; ${ }^{3}$ Fatih University Medical Faculty, Ankara, Turkey

Epigenetic mechanisms might play an important role in development of BPD. The aim of this study was to evaluate the protective effect of valproic acid (VPA), an histon deacetylase inhibitor, in hyperoxic lung injury in neonatal rat model.

Methods A total of 30 rat pups (0 days old) were divided equally into 3 groups: control, hyperoxia and hyperoxia+VPA groups. In hyperoxia groups, pups were maintained in $95 \% \mathrm{O}_{2}$ for 10 days while control group was maintained in room air. VPA was administered intraperitoneally once daily for the first 10 days of life. On day 10, histopathological score, radial alveolar count, lamellar protein count, histone deacetylase activity (HDAC), proinflammatory cytokine concentrations were determined with ELISA, whereas acetylated $\mathrm{H} 4$ protein and caspase- 3 expression were evaluated with Western-Blot analysis. Also apoptosis was evaluated with TUNEL method.

Results The histopathological score, radial alveolar count, lamellar protein count of the pups in VA group were significantly higher. VPA also preserved alveolarization significantly and fibrosis was significantly decreased in rat pups exposed to VPA treatment. HDAC activity significantly reduced with VPA treatment. The proinflammatory markers, caspase- 3 expression and number of TUNEL positive cells were also significantly decreased with VPA treatment. Acetylated $\mathrm{H} 4$ protein expression was significantly higher in the hyperoxia+VPA group.

Conclusion All these data suggest that VPA might provide possible protective effect against hyperoxic lung injury as an histone deacetylase inhibitor. VPA exhibit these effects by preserving alveolarization, decreasing fibrosis and inflammation via decreasing $\mathrm{HDAC}$ activity, increasing acetylated $\mathrm{H} 4$ protein expression and reducing inflammation.

\section{EFFECTS OF CATALYTIC ANTIOXIDANT MNTBAP ON PULMONARY ANGIOGENIC AND OXIDATIVE GENE EXPRESSION TO HYPEROXIA IN NEWBORN MICE}

doi:10.1136/archdischild-2012-302724.0598

1,2B Paturi, ${ }^{2} \mathrm{RM}$ Ryan, ${ }^{2} \mathrm{~L}$ Nielson, ${ }^{2} \mathrm{H}$ Wang, ${ }^{2} \mathrm{~V}$ Kumar. ${ }^{1}$ Neonatology, Rotunda Hospital, Dublin, Ireland; '2Neonatology, WCHOB, SUNY, Buffalo, NY, USA

Background Development of lung injury during prolonged $\mathrm{O}_{2}$ exposure is a complex process, associated with changes in expression of a number of genes important in the adaptive response to hyperoxia. MnTBAP is a compound with strong antioxidant properties.

Objective To study the effects of MnTBAP on angiogenic and oxidative gene expression in C57BL6 neonatal mice following hyperoxia.

Design and methods: Newborn mice litters were randomized on postnatal day 4 to hyperoxia $\left(>95 \% \mathrm{O}_{2}\right)(\mathrm{OX})$ or room air (RA) for $72 \mathrm{hrs}$ during which they received MnTBAP $(\mathrm{MN}) 10 \mathrm{mg} / \mathrm{kg}$ or saline (SL) daily by IP injection for 3 days and then were sacrificed. Whole lung angiogenic and oxidative gene expression profiling (84 related genes for each) was done by real-time, reverse transcription, quantitative PCR ( $n=4)$. Data was processed and analyzed using SA Biosciences PCR array data analysis web portal.

Results Hyperoxia significantly upregulated peroxiredoxin 6 expression compared to room air exposed newborn mice. Treatment with MnTBAP downregulated the expression of myeloperoxidase and Prdx6-rs1. Hyperoxia downregulated the expression of angiogenic genes such as angiopoietin $1 \& 2$, TGF 1, TGF 3 and HGF; MnTBAP treatment during the hyperoxia exposure reversed this effect and these genes were upregulated.

Conclusions The catalytic antioxidant MnTBAP reversed the effects of hyperoxia on angiogenic gene expression in newborn mice. The protective effects of antioxidants in newborn hyperoxia models need to be studied further to provide additional understanding of the management of bronchopulmonary dysplasia.

\section{CARDIOVASCULAR CONSEQUENCES OF BRONCHOPULMONARY DYSPLASIA IN PREMATURELY BORN PRESCHOOL CHILDREN}

doi:10.1136/archdischild-2012-302724.0599 